\title{
Quasi-Maximum Likelihood Estimation of Long-Memory Stochastic Volatility Models*
}

Rosemeire O. Ferraz ${ }^{* *}$

Luiz K. Hotta ${ }^{* * *}$

\begin{abstract}
We analyze finite sample properties of the quasi-maximum likelihood estimators of longmemory stochastic volatility models. The estimates are done in the time domain using autoregressive and moving average in the state space representation. The results are compared with usual estimators of the long-memory parameter.
\end{abstract}

Keywords: Fractional Integration, Stochastic Volatility, Quasi-Maximum Likelihood.

JEL Codes: C13, C22.

\footnotetext{
${ }^{*}$ Submitted in July 2006. Revised in July 2007. This work was partially supported by grants from CNPq, CAPES and FAPESP. We also thank Laboratório Epifisma and comments from M. Zavallos and two anonymous referees.

${ }^{* *}$ Fundação Centro Médico de Campinas.

*** Department of Statistics, State University of Campinas. E-mail: hotta@ime.unicamp.br

Brazilian Review of Econometrics

v. $27, \mathrm{n}^{\circ} 2$, pp. $225-233 \quad$ November 2007
} 


\section{Introduction}

A typical financial time series of returns is characterized by the so-called stylized facts, i.e., excess kurtosis, volatility clustering and almost no serial correlation in the level, but with a persistent serial correlation in the squares of the series. In some series, this decay can be very slow, suggesting that the squared returns have a long-memory characteristic (see, for instance, Ding et al. (1993)). Harvey (1998) and Breidt et al. (1998) extended the stochastic volatility model to the long-memory stochastic volatility (LMSV) class of models in order to incorporate this long memory. These models have found application in many financial series (see, for instance, Chan and Petris (2000), Crato and Ray (2002), Pérez and Ruiz (2001a), and Deo et al. (2006)). A review of the long-memory parameter estimation of LMSV models can be found in Pérez and Ruiz (2001a) and in Crato and Ray (2002). Harvey (1998) and Breidt et al. (1998) proposed an estimation method based on the spectral approximation to the Gaussian likelihood, and the finite sample properties of this estimator were analyzed by Pérez and Ruiz (2001b). Arteche (2004) analyzed the performance of the Gaussian semiparametric or local Whittle estimator, and Jensen (2004) suggested a Bayesian semiparametric method. A survey on the estimation of long-memory time series models based on likelihood can be found in Chan and Palma (2006).

The approach adopted here is based on the state space representation of the truncated ARFIMA process. The approximation of the ARFIMA model by truncated state space representation was analyzed by Chan and Palma (1998), Basak et al. (2001) and Palma (2007, Ch. 4). Chan and Petris (2000) suggested applying a Bayesian MCMC-based approach to this approximated model. On the other hand, the LMSV model can be written as an ARFIMA model with non-Gaussian disturbances. Therefore, we can approximate the non-Gaussian ARFIMA model by truncated state space representation and evaluate the (approximated) quasilikelihood directly by the Kalman filter algorithm. The quasi-maximum likelihood (QML) estimate can be obtained using an appropriate maximization algorithm. Pérez and Ruiz (2001b) commented that the small sample properties of the QML estimator of the LMSV model were not known yet. In this paper, we use simulation to investigate the small sample properties of this estimator, the choice of the truncation order and the MA and AR representation. We also compare the properties of the QML estimator with two traditional methods based on the frequency domain, the GPH (Geweke and Porter-Hudak, 1983) and the GSP (Gaussian semiparametric estimator of Robinson (1995)).

This paper is organized as follows. Section 2 introduces the state space approach and its application to LMSV models. It also introduces the AR and MA representation and the QML estimator. Section 3 presents the two estimators used in the comparison. Section 4 presents the simulation, and concluding remarks are given in Section 5. 


\section{State Space Representation and the QML Estimator}

The simplest LMSV process can be written as:

$$
\begin{aligned}
y_{t} & =\sigma e^{h_{t} / 2} \epsilon_{t} \\
(1-B)^{d} h_{t} & =\eta_{t}
\end{aligned}
$$

where $\epsilon_{t}$ is a standard Gaussian process, $\sigma e^{h_{t} / 2}$ denotes the conditional volatility of the series, $n_{t}$ is $N\left(0, \sigma_{\eta}^{2}\right),\left\{\epsilon_{t}\right\}$ and $\left\{\eta_{t}\right\}$ are white noise independent processes, $B$ is the backshift operator and $d \in(0,0.5)$. We only consider the fractional parameter in this region because, in empirical analysis, we find estimation in this region and we do not expect return series with an explosive behavior. Denote this model as SV-ARFIMA $(0, d, 0)$.

Model (1-2) can be linearized as:

$$
z_{t}=\mu+h_{t}+\xi_{t}
$$

where $z_{t}=\log \left(y_{t}^{2}\right), \mu=\log \left(\sigma^{2}\right)+E\left(\log \left(\epsilon_{t}^{2}\right)\right)$ and $\xi_{t}=\log \left(\epsilon_{t}^{2}\right)-E\left(\log \left(\epsilon_{t}^{2}\right)\right)$. Therefore, $\left\{\xi_{t}\right\}$ is a white noise process and we have a state space representation when the $\operatorname{ARFIMA}(0, d, 0)$ process is approximated as a truncated ARMA process.

We propose two types of approximation. In the autoregressive (AR) approximation we have

$$
\sum_{i=0}^{P} \Pi_{i} h_{t-i}=\eta_{t}
$$

with $\Pi_{0}=1$ and $\Pi_{j}=[(j-d-1) / j] \Pi_{j-1}, j \geq 1$.

In the MA representation, the log-volatility is written as:

$$
(1-B) h_{t}=(1-B)^{1-d} \eta_{t}=\sum_{i=0}^{Q} \Theta_{i} \eta_{t-i}
$$

with $\Theta_{0}=1$ and $\Theta_{j}=[(j+d-1) / j] \Theta_{j-1}, j \geq 1$.

Equations (2.3) and (2.4) provide the AR state space representation of the LMSV model, while equations (2.3) and (2.5) provide the MA representation. By applying the Kalman filter under a Gaussian assumption, we have an approximated QML estimator. Chan and Palma (1998) suggested using the MA representation because the coefficients $\Theta_{j}$ converge to zero faster than the AR representation coefficients, allowing for a smaller truncation order. However, differently from Chan and Palma (1998), we do not take the difference in the return series, we keep the level of the log-volatility in the space vector. In their work, they presented simulation results for $d$ values ranging from 0.05 to 0.40 and $m=6$ for sample size $100, m=14$ for sample size 10,000 and $m=30$ for sample size 1,000 . In financial 
applications, the usual sample size is around 1,000 to 10,000. Chan and Petris (2000) also used MA representation for 28 years of daily returns and found that $Q$ values between 10 and 20 provide a sufficiently accurate approximation.

An ARMA representation is theoretically possible, but it is not used because the representation is not unique and we would lose the main advantage of the $\mathrm{AR}$ and MA approximation, which is the easy implementation of the Kalman recursion.

\section{Traditional Estimation Procedures}

Since the LMSV process can be written as an ARFIMA process, the fractional parameter can be estimated by traditional methods. We selected two of these commonly used traditional methods to compare with our QML estimators. The selected estimators are presented briefly in what follows.

\subsection{The spectral regression method - GPH}

The GPH periodogram-based method proposed by Geweke and Porter-Hudak (1983) is largely used to estimate an ARFIMA model without additive white noise. They suggested regressing the log of the estimated spectrum of the series on the $\log$ of the frequency argument. They also suggested using only frequencies close to zero in order to avoid the contamination of short-memory correlation. After this work, many suggestions appeared in the literature, either using different spectrum estimators or frequency selection. In LMSV, the linearized model is in fact an ARFIMA process with white noise. In this case, Deo and Hurvich (2001) showed that the GPH estimator is negatively biased. Crato and Ray (2002) showed that preliminary smoothing and aggregation can improve the GPH method. In the comparison, however, we use the original GPH estimator because it is already implemented in many statistical programs and no modification performs remarkably better than the other in all situations. We consider the number of periodogram ordinates $m=n^{0.5}$.

\subsection{Gaussian semiparametric method - GSP}

The GSP method was proposed by Robinson (1995). He considered that the negative of the log-likelihood function of a $\operatorname{Gaussian} \operatorname{ARFIMA}(p, d, q)$ process, denoted by $L$, can be approximated by:

$$
L \approx \frac{1}{m}\left[\sum_{j=1}^{m} \frac{I_{n}\left(\lambda_{j}\right)}{c \lambda_{j}^{-2 d}}+\sum_{j=1}^{m} \log \left(c \lambda_{j}^{-2 d}\right)\right]
$$

in a neighborhood of zero frequencies delimited by $m$, where $\lambda_{j}$ is the Fourier frequency. An estimate of $d$ is obtained by $m$, where $\lambda_{j}$ is the Fourier frequency. An estimate of $d$ is obtained by minimizing L. Similarly to the GPH, the added 
noise will cause bias in the estimation of $d$. However, for the same reason, we will consider this estimator in the comparison process. We consider the number of periodogram ordinates $m=n^{0.5}$.

\section{Simulation Study}

The performance of the estimators are studied via simulation. We considered the $\operatorname{ARFIMA}(0, d, 0)$ and $\operatorname{ARFIMA}(1, d, 0)$ with $d=0.20,0.30,0.40$ and $0.45, \phi=$ 0.7 and 0.9 , sample size equal to $1000, \sigma_{\eta}^{2}=1.0$ and $\sigma_{\epsilon}^{2}=1.0$. The latent ARFIMA processes were generated using S-Plus and the estimation program was written in Ox. The GPH and GSP estimates were found using an Ox program available in Doornik (1999). For some series, we have a convergence problem. The results presented for all the estimators are for the series without a convergence problem.

\subsection{ARFIMA $(0, \mathrm{~d}, 0)$}

For this process, we used 300 replications. We first compare the estimators of the long-memory parameter using AR and MA approximations with truncation orders equal to 20 and 30, which give a total of four estimators. Table 1 presents the sample variances of these QML estimators in the diagonal and the correlations of the estimates in the upper part of the matrix. The number of problems in the convergence is given in the 2 nd and 7 th columns. The percentage was always small and decreased when d increased.

Table 1

Correlation among the QML estimates of parameter $d$ in the $\operatorname{ARFIMA}(0, d, 0)$ model. Variances in the diagonal and number of nonconvergence in the 2nd and 7th columns

\begin{tabular}{|c|c|c|c|c|c|c|c|c|c|c|}
\hline \multirow{2}{*}{$\begin{array}{l}\text { estimator } \\
\text { AR20 }\end{array}$} & \multirow{2}{*}{$\begin{array}{c}\begin{array}{c}\text { non } \\
\text { conv. }\end{array} \\
2\end{array}$} & \multicolumn{4}{|c|}{$\mathrm{d}=0.20$} & \multirow{2}{*}{$\begin{array}{c}\begin{array}{c}\text { non } \\
\text { conv. }\end{array} \\
7\end{array}$} & \multicolumn{4}{|c|}{$\mathrm{d}=0.30$} \\
\hline & & 0.035 & 0.823 & 0.550 & 0.523 & & .036 & 0.921 & 0.795 & $\overline{0.841}$ \\
\hline AR30 & 4 & & 0.035 & 0.551 & 0.594 & 4 & & 0.025 & 0.800 & 0.825 \\
\hline MA20 & 10 & & & 0.015 & 0.903 & 9 & & & 0.019 & 0.944 \\
\hline \multirow[t]{2}{*}{ MA30 } & 10 & & & & 0.019 & 5 & & & & $\overline{0.022}$ \\
\hline & & \multicolumn{4}{|c|}{$\mathrm{d}=0.40$} & & \multicolumn{4}{|c|}{$\mathrm{d}=0.45$} \\
\hline AR20 & 2 & 0.032 & 0.965 & 0.758 & 0.802 & 1 & 0.028 & 0.978 & 0.789 & 0.821 \\
\hline AR30 & 1 & & 0.024 & 0.779 & 0.835 & 1 & & 0.022 & 0.806 & $\overline{0.839}$ \\
\hline MA20 & 2 & & & 0.020 & 0.976 & 1 & & & 0.020 & 0.979 \\
\hline MA30 & 1 & & & & 0.022 & 1 & & & & 0.021 \\
\hline
\end{tabular}

In general, the correlations were larger between MA20 and MA30 than between AR20 and AR30 estimates and increased with $d$. The correlations between AR20 and AR30 and between MA20 and MA30 were almost equal to one for $d$ larger than or equal to 0.30 , indicating that, in these cases, there is little difference in using truncations equal to 20 or 30 . For this reason, Table 2 presents only the comparison (bias, standard deviation and mean square error (MSE)) among the AR30, MA30, GPH and GSP estimates. 
The MA30 estimator presented the smallest bias in all cases, except when $d=$ 0.4, when it was second to the AR30 estimator. The GSP estimator presented the smallest standard deviation, but the MA30 yielded the best overall performance in terms of MSE. Generally, the QML estimator is better than the GPH and GSP in terms of bias and MSE, and the performances of MA30 and AR30 were very close for $d \geq 0.30$.

Table 2

Comparison of the estimation of the $\operatorname{ARFIMA}(0, d, 0)-\mathrm{SV}$ process - the best performance is in boldface

\begin{tabular}{|c|c|c|c|c|c|c|c|c|}
\hline \multirow[t]{2}{*}{$\mathrm{d}$} & \multicolumn{4}{|c|}{ bias } & \multicolumn{4}{|c|}{$\mathrm{sd}$} \\
\hline & AR30 & MA30 & GPH & GSP & AR30 & MA30 & GPH & GSP \\
\hline 0.20 & -0.088 & -0.043 & -0.110 & -0.123 & 0.187 & 0.139 & 0.143 & 0.117 \\
\hline 0.30 & -0.064 & -0.045 & -0.125 & -0.140 & 0.159 & 0.148 & 0.137 & 0.116 \\
\hline 0.40 & -0.037 & -0.038 & -0.109 & -0.127 & 0.154 & 0.149 & 0.138 & 0.116 \\
\hline 0.45 & -0.024 & -0.023 & -0.119 & -0.124 & 0.147 & 0.146 & 0.154 & 0.125 \\
\hline \multirow[t]{2}{*}{ d } & \multicolumn{4}{|c|}{ MSE } & & & & \\
\hline & AR30 & MA30 & $\mathrm{GPH}$ & GSP & & & & \\
\hline 0.20 & 0.043 & 0.021 & 0.032 & 0.029 & & & & \\
\hline 0.30 & 0.030 & 0.024 & 0.035 & 0.033 & & & & \\
\hline 0.40 & 0.025 & 0.024 & 0.031 & 0.030 & & & & \\
\hline 0.45 & 0.022 & 0.022 & 0.038 & 0.031 & & & & \\
\hline
\end{tabular}

\section{2 $\operatorname{ARFIMA}(1, \mathrm{~d}, 0)$}

For this process, we used 160 replications. Initially, the comparison among the four QML estimators is presented in Table 3. We present only the results for the estimation of $d$ and $\phi$ and not for the variances. As to the estimation of $d$, the MA30 generally had the best performance, but the differences were small when $\phi=0.9$. The biases decreased considerably compared to the $\operatorname{ARFIMA}(0, d, 0)$ case, except when $d$ was large and $\phi=0.9$. In this case, the biases were almost the same. Although the correlations are not presented, the correlations between the MA estimators are larger than between the AR estimators. The correlation decreases when $\phi$ increases, but up to $\phi=0.70$, the correlations were close to the case when $\phi=0$. However, the correlation decreased considerably for $\phi=0.9$, especially for large $d$. For instance, the correlation between MA30 and MA20, for $d=0.45$ was equal to 0.724. As to the estimation of $\phi$, the AR30 estimator generally had the best performance, but there was almost no significant difference when $\phi=0.9$.

Table 4 presents the comparison among the AR30, MA30, GPH and GSP estimators for the estimation of $d$. The bias of the GPH and GSP estimators also decreased when $\phi=0.70$, but increased when $\phi=0.90$. When $\phi=0.70$, the GSP estimator generally presented the smallest MSE, except when $d=0.20$, where the MSE of the MA30 estimator was smaller, and the AR30 estimator presented the worst result. However, when $\phi=0.90$, the GSP and GPH presented the worst results in terms of MSE, whereas the MA30 had the best ones. 
Table 3

Comparison of the QML estimation of $d$ and $\phi$ in the ARFIMA $(1, d, 0)$-SV process the best performance is in boldface

\begin{tabular}{|c|c|c|c|c|c|c|c|}
\hline \multirow[t]{3}{*}{$\mathrm{d}$} & \multirow[t]{3}{*}{ estimator } & \multicolumn{6}{|c|}{$\phi=0.7$} \\
\hline & & & $\bar{d}$ & & & $\bar{\phi}$ & \\
\hline & & bias & sd & MSE & bias & sd & MSE \\
\hline \multirow{4}{*}{0.20} & AR20 & -0.055 & 0.205 & 0.045 & -0.083 & 0.261 & 0.075 \\
\hline & AR30 & -0.088 & 0.222 & 0.057 & -0.046 & 0.237 & 0.058 \\
\hline & MA20 & -0.046 & 0.123 & 0.017 & -0.038 & 0.213 & 0.047 \\
\hline & MA30 & -0.010 & 0.118 & 0.014 & -0.092 & 0.248 & 0.070 \\
\hline \multirow{4}{*}{0.30} & AR20 & -0.025 & 0.200 & 0.041 & -0.072 & 0.225 & 0.056 \\
\hline & AR30 & -0.052 & 0.191 & 0.039 & -0.039 & 0.207 & 0.044 \\
\hline & MA20 & -0.053 & 0.167 & 0.031 & -0.025 & 0.221 & 0.050 \\
\hline & MA30 & -0.021 & 0.161 & 0.027 & -0.059 & 0.230 & 0.056 \\
\hline \multirow{4}{*}{0.40} & AR20 & 0.016 & 0.179 & 0.033 & -0.159 & 0.268 & 0.097 \\
\hline & AR30 & -0.032 & 0.196 & 0.040 & -0.083 & 0.222 & 0.056 \\
\hline & MA20 & -0.052 & 0.184 & 0.037 & -0.055 & 0.235 & 0.058 \\
\hline & MA30 & -0.012 & 0.163 & 0.027 & -0.099 & 0.248 & 0.071 \\
\hline \multirow{4}{*}{0.45} & AR20 & 0.043 & 0.195 & 0.040 & -0.164 & 0.263 & 0.096 \\
\hline & AR30 & -0.002 & 0.202 & 0.041 & -0.106 & 0.236 & 0.067 \\
\hline & MA20 & -0.042 & 0.198 & 0.041 & -0.075 & 0.264 & 0.075 \\
\hline & MA30 & -0.009 & 0.180 & 0.032 & -0.099 & 0.254 & 0.074 \\
\hline \multirow[t]{3}{*}{$\bar{d}$} & esti & \multicolumn{6}{|c|}{$\phi=0.9$} \\
\hline & ma & \multicolumn{3}{|c|}{$d$} & \multicolumn{3}{|c|}{$\phi$} \\
\hline & tor & bias & sd & MSE & bias & sd & MSE \\
\hline \multirow{4}{*}{0.20} & AR20 & -0.016 & 0.224 & 0.051 & -0.048 & 0.173 & 0.032 \\
\hline & AR30 & -0.005 & 0.209 & 0.044 & -0.041 & 0.134 & 0.020 \\
\hline & MA20 & -0.036 & 0.175 & 0.032 & -0.029 & 0.170 & 0.030 \\
\hline & MA30 & 0.009 & 0.183 & 0.034 & -0.046 & 0.174 & 0.032 \\
\hline \multirow{4}{*}{0.30} & AR20 & -0.006 & 0.231 & 0.054 & -0.029 & 0.104 & 0.012 \\
\hline & AR30 & 0.000 & 0.209 & 0.044 & -0.026 & 0.091 & 0.009 \\
\hline & MA20 & -0.065 & 0.180 & 0.037 & 0.004 & 0.074 & 0.006 \\
\hline & MA30 & -0.007 & 0.189 & 0.036 & -0.015 & 0.081 & 0.007 \\
\hline \multirow{4}{*}{0.40} & AR20 & 0.024 & 0.278 & 0.078 & -0.063 & 0.131 & 0.021 \\
\hline & AR30 & 0.049 & 0.244 & 0.062 & -0.062 & 0.112 & 0.016 \\
\hline & MA20 & 0.013 & 0.252 & 0.064 & -0.053 & 0.151 & 0.026 \\
\hline & MA30 & 0.038 & 0.229 & 0.054 & -0.057 & 0.142 & 0.023 \\
\hline \multirow{4}{*}{0.45} & AR20 & -0.036 & 0.306 & 0.095 & -0.037 & 0.136 & 0.020 \\
\hline & AR30 & 0.035 & 0.280 & 0.080 & -0.062 & 0.146 & 0.025 \\
\hline & MA20 & 0.058 & 0.265 & 0.074 & -0.086 & 0.214 & 0.053 \\
\hline & MA30 & 0.074 & 0.228 & 0.058 & -0.073 & 0.161 & 0.031 \\
\hline
\end{tabular}


Table 4

Comparison of the estimation of $\mathrm{d}$ in the $\operatorname{ARFIMA}(1, d, 0)-\mathrm{SV}$ process - the best performance is in boldface

\begin{tabular}{|c|c|c|c|c|c|c|c|c|c|}
\hline \multirow[t]{2}{*}{$\mathrm{d}$} & \multirow[t]{2}{*}{$\phi$} & \multicolumn{4}{|c|}{ bias } & \multicolumn{4}{|c|}{$\mathrm{sd}$} \\
\hline & & AR30 & MA30 & GPH & GSP & AR30 & MA30 & $\mathrm{GPH}$ & GSP \\
\hline \multirow[t]{2}{*}{0.20} & 0.7 & -0.088 & -0.010 & 0.001 & -0.002 & 0.222 & 0.118 & 0.151 & 0.128 \\
\hline & 0.9 & -0.005 & 0.009 & 0.229 & 0.239 & 0.209 & 0.183 & 0.154 & 0.134 \\
\hline \multirow[t]{2}{*}{0.30} & 0.7 & -0.052 & -0.021 & 0.001 & -0.004 & 0.191 & 0.161 & 0.148 & 0.135 \\
\hline & 0.9 & 0.000 & -0.008 & 0.228 & 0.235 & 0.209 & 0.189 & 0.150 & 0.149 \\
\hline \multirow[t]{2}{*}{0.40} & 0.7 & -0.033 & -0.012 & 0.023 & 0.018 & 0.196 & 0.163 & 0.137 & 0.123 \\
\hline & 0.9 & 0.048 & 0.037 & 0.252 & 0.256 & 0.244 & 0.229 & 0.138 & 0.127 \\
\hline \multirow[t]{2}{*}{0.45} & 0.7 & -0.002 & -0.009 & 0.035 & 0.026 & 0.202 & 0.180 & 0.152 & 0.132 \\
\hline & 0.9 & 0.034 & 0.073 & 0.256 & 0.257 & 0.280 & 0.228 & 0.143 & 0.131 \\
\hline \multirow[t]{2}{*}{$\mathrm{d}$} & $\phi$ & \multicolumn{4}{|c|}{ MSE } & & & & \\
\hline & & AR30 & MA30 & $\mathrm{GPH}$ & GSP & & & & \\
\hline \multirow[t]{2}{*}{0.20} & 0.7 & 0.057 & 0.014 & 0.023 & 0.017 & & & & \\
\hline & 0.9 & 0.044 & 0.034 & 0.076 & 0.075 & & & & \\
\hline \multirow[t]{2}{*}{0.30} & 0.7 & 0.039 & 0.027 & 0.022 & 0.018 & & & & \\
\hline & 0.9 & 0.044 & 0.036 & 0.075 & 0.078 & & & & \\
\hline \multirow[t]{2}{*}{0.40} & 0.7 & 0.040 & 0.027 & 0.019 & 0.016 & & & & \\
\hline & 0.9 & 0.062 & 0.054 & 0.083 & 0.082 & & & & \\
\hline \multirow[t]{2}{*}{0.45} & 0.7 & 0.041 & 0.032 & 0.024 & 0.018 & & & & \\
\hline & 0.9 & 0.080 & 0.058 & 0.086 & 0.084 & & & & \\
\hline
\end{tabular}

\section{Conclusions}

The quasi-maximum likelihood approach has the advantage of estimating all the parameters simultaneously and also providing the estimates of the volatility, the state in the Kalman filter. The QML performed better at estimating the long-memory parameter than did traditional estimators. Among the four QML estimators tested, we recommend using the MA30 estimator because it had the best performance in most of the cases or was closest to the best in the other cases.

\section{References}

Arteche, J. (2004). Gaussian semiparametric estimation in long memory in stochastic volatility and signal plus noise models. Journal of Econometrics, 119:131154.

Basak, G. K., Chan, N. H., \& Palma, W. (2001). On the approximation of long memory processes by an ARMA model. Journal of Forecasting, 20:367-389.

Breidt, F. J., Crato, N., \& de Lima, P. J. F. (1998). The detection and estimation of long-memory in stochastic in stochastic volatility. Journal of Econometrics, $83: 325-348$.

Chan, N. H. \& Palma, W. (1998). State space modelling of long memory processes. Annals of Statistics, 26:719-740.

Chan, N. H. \& Palma, W. (2006). Estimation of long-memory time series models: A survey of different likelihood-based methods. In Fomby, T. B. \& Terrel, D., 
editors, Econometric Analysis of Financial and Economic Time Series, pages 89-121. Elsevier, Amsterdam. Advanced Econometrics.

Chan, N. H. \& Petris, G. (2000). Lonf memory stochastic volatility: A Bayesian approach. Communication in Statistics - Theory and Methods, 29:1367-1378.

Crato, N. \& Ray, B. K. (2002). Semi-parametric smoothing estimators for long memory processes with added noise. Journal of Statistical Planning and Inference, 105:283-297.

Deo, R., Hurvich, C., \& Lu, Y. (2006). Forecasting realized volatility using a long memory stochastic volatility model: Estimation, prediction and seasonal adjustment. Journal of Econometrics, 131:29-58.

Deo, R. \& Hurvich, C. M. (2001). On the log-periodogram regression estimator of memory parameter in long memory stochastic volatility models. Econometric Theory, 17:686-710.

Ding, Z., Granger, C. W. J., \& Engle, R. F. (1993). A long memory property of stock market returns and a new model. Journal of Empirical Finance, 1:83-106.

Doornik, J. A. (1999). Object-Oriented Matrix Programming Using Ox 2.1. Timberlake Consultants Ltd.

Geweke, J. \& Porter-Hudak, S. (1983). The estimation and application of longmemory time series models. Journal of Time Series Models, 4:221-237.

Harvey, A. C. (1998). Long memory in stochastic volatility. In Knight, J. \& Satchell, E., editors, Forecasting Volatility in Financial Markets, pages 307-320. Butterworth-Haineman, London.

Jensen, M. J. (2004). Semiparametric Bayesian inference of long-memory stochastic volatility models. Journal of Time Series Analysis, 25:895-922.

Palma, W. (2007). Long-Memory Time Series: Theory and Methods. WileyInterscience, Hoboken.

Pérez, A. \& Ruiz, E. (2001a). Finite sample properties of a QML estimator of stochastic volatility models with long memory. Economics Letters, 70:157-164.

Pérez, A. \& Ruiz, E. (2001b). Modelos de memoria larga para series económicas y financeiras. Documento de Trabajo 01-01, Serie de Estadística y Econometría, Universidad Carlos III de Madri.

Robinson, P. M. (1995). Gaussian semiparametric estimation of long range dependence. Annals of Statistics, 23:1630-1661. 